\title{
Relationship between negative symptoms and neurocognitive functions in adolescent and adult patients with first-episode schizophrenia
}

Manli Huang ${ }^{1 \dagger}$, Yi Huang ${ }^{2 \dagger}$, Liang $\mathrm{Yu}^{3}$, Jianbo Hu${ }^{1}$, Jinkai Chen ${ }^{1}$, Pingbo Jin ${ }^{1}$, Weijuan $\mathrm{Xu}^{1}$, Ning Wei ${ }^{1}$, Shaohua Hu${ }^{1}$, Hongli Qi ${ }^{1}$ and Yi Xu ${ }^{1 *}$

\begin{abstract}
Background: This study aimed to explore differences in links between negative symptoms and neurocognitive deficits in adolescent and adult patients with first-episode schizophrenia. Schizophrenia is a mental disorder often characterized by positive and negative symptoms, reduced emotional expression, excitatory status, and poor cognitive ability. The severity of negative symptoms in patients with schizophrenia was reported to be more related to poor quality of life, weak functional ability, and heavy burden from families than with the severity of positive symptoms. Previous studies suggested correlations between the severity of negative symptoms in patients with schizophrenia and neurocognitive deficits.

Methods: This study included 92 patients (33 adolescents and 59 adults) with first-episode schizophrenia and 57 healthy people matched by age and education level. Neurocognitive functions and clinical symptoms were assessed using a standardized questionnaire.

Results: Patients with first-episode schizophrenia showed neurocognitive deficits in most neuropsychological assessments compared with healthy people. With the variable of education level controlled, the negative factor score of adolescent patients with first-episode schizophrenia was strongly correlated with more time spent in part $1(r=.646)$ and part $2(r=.663)$ of the trail making test, and moderately correlated to more perseverative errors $(r=.425)$ of the Wisconsin card sorting test and fewer correct trials $2(r=-.425)$ of the continuous performance test. However, no such correlations were found in adult patients.
\end{abstract}

Conclusions: This study indicated significant correlations between negative symptoms and most neurocognitive functions in patients with first-episode schizophrenia, with a stronger correlation in adolescent patients.

Trial registration: The trial registration number is ChiCTR-COC-14005302, while retrospectively registered on January 5, 2014.

Keywords: Adolescent patients with first-episode schizophrenia, Correlation, Negative symptoms, Neurocognitive functions, PANSS

\footnotetext{
* Correspondence: yixu1961@163.com

${ }^{\dagger}$ Equal contributors

${ }^{1}$ Department of Psychiatry, The First Affiliated Hospital, College of Medicine,

Zhejiang University, The Key Laboratory of Mental Disorder's Management of

Zhejiang Province, No. 79, Qingchun Road, Hangzhou 310003, China

Full list of author information is available at the end of the article
} 


\section{Background}

Schizophrenia is a mental disorder often described in terms of hallucination and abnormal social behavior, including poor rapport and social isolation. It affects about $0.3-0.7 \%$ [1] of people at some point (the peak age of onset is 18-25 years [2]) in their lives and accounts for approximately $1 \%$ of disability-adjusted life year all over the world [3]. Patients with schizophrenia have been reported to have abnormities in brain structure and function [1, 4]. The causes of schizophrenia have been widely investigated, and many studies have suggested genetics, prenatal and adolescent neurodevelopment, psychological health, and social living environment as common risk factors $[2,5,6]$.

Schizophrenia is often characterized by positive and negative symptoms, reduced emotional expression, excitatory status, and poor cognitive ability. The positive and negative syndrome scale (PANSS) is a 30-item rating scale designed by Kay to assess the severity of schizophrenia symptoms. Literature research suggested that a five-factor model, which consists of positive, negative, disorganized-concrete, excited, and anxious-depressive factor, better captured the PANSS structure in patients with schizophrenia [7-14].

Generally, negative symptoms refer to poor emotional reactions or thought processes, including emotion impoverishment, speech barrier, thought inflexibility, and decreased activity. It has been reported that severity of negative symptoms in patients with schizophrenia was more related to poor quality of life [15], weak functional ability [16] and heavy burden from families, compared with the severity of positive symptoms [17]. Moreover, patients with schizophrenia having obvious negative symptoms often had a history of poor social adaptability before the onset of illness, and their responses to medication were often limited [18]. The present study focused on negative symptoms owing to their serious influence on human life.

Adolescence is a key period of neurological and psychological development. If psychosis occurs in one's late adolescence when the frontal lobe is still developing, some extraordinary differences may exist in both neurocognitive deficits and clinical symptoms [19], compared with an adult patient with schizophrenia. A 5-year followup clinical study on very early-onset schizophrenia, using magnetic resonance imaging (MRI), showed damage processes in brain regions of adolescent patients with schizophrenia. However, adult cognitive deficits are known to be mediated by environmental factors [20]. A latest study also showed that adolescent patients with schizophrenia performed worse than adult patients in tasks of working memory, language, and motor function [21].

In addition, previous studies have suggested correlations between negative symptoms in patients with schizophrenia and neurocognitive deficits involving intelligence, executive function, sustained-attention function, sensory motor function [22], cognitive function [23], and memory [22, 24]. Patients with higher negative scores of PANSS had more perseverative errors (PE) and fewer completed categories (CC) in the Wisconsin card sorting test (WCST) (standing for executive function) [25], and poorer performance in University of Pennsylvania Smell Identification Test (standing for function of an individual's olfactory system) [26]. They also experienced more difficulties in the trail making test (TMT) (standing for visual attention and executive function), verbal fluency test (standing for semantic memory) [25], and faux pas test (standing for sociability) [27].

As mentioned, correlations exist between negative symptoms in patients with schizophrenia and neurocognitive deficits. To get rid of clinical interference, we aimed to patients with first-episode schizophrenia who were drug naive. Thomas R. Insel pointed out that schizophrenia should be regarded a collection of neurodevelopmental disorders [2]. Since both symptoms and neurocognitive deficits may be caused by neurodevelopmental problems in adolescent patients with first-episode schizophrenia whose prefrontal cortices are under development [28-30], it is assumed that their negative symptoms are more closely related to neurocognitive deficits than those of adult patients with first-episode schizophrenia having developed prefrontal cortices. If the aforementioned hypothesis is correct, the negative symptom scores of adolescent patients with schizophrenia become more important in evaluating neurocognitive deficits, compared with adult patients with first-episode schizophrenia. It is easy for psychiatrists to obtain patients' PANSS scores, while evaluation of neurocognitive functions is complicated and time-consuming. That is to say, lower negative symptom scores indicate stronger neurocognitive deficits in adolescent patients with schizophrenia. In addition, improvement of patients' negative symptoms can be used in evaluating the therapeutic effect.

The present study had two objectives (the first objective was replication of the objective of previous studies to some degree, while the second objective was novel):

1. To evaluate neurocognitive deficits and clinical symptoms of patients with first-episode schizophrenia.

2. To study the relationship between negative symptoms and neurocognitive functions in patients with first-episode schizophrenia, especially adolescent patients.

\section{Methods}

\section{Study subjects}

This study included 92 patients with first-episode schizophrenia and 57 healthy volunteers. All the patients were divided into two groups-33 adolescent patients with first-episode schizophrenia (whose age and age of onset 
were less than 18 years) and 59 adult patients with firstepisode schizophrenia (whose age and age of onset were equal to or more than 18 years).

Patients were recruited from the First Affiliated Hospital of Medicine School of Zhejiang University, who fulfilled diagnostic criteria for schizophrenia in the Diagnostic and Statistical Manual of Mental Disorders, Fourth Edition (DSM-IV). The Structured Clinical Interview for DSM-IVTR, routine laboratory tests, and physical and neurological examinations were all administered to each participant.

Inclusion criteria for patient selection were as follows: (1) age between 13 and 45 years; (2) having a DSM-IV diagnosis of schizophrenia; (3) experiencing their first episode; (4) being drug naive; (5) both males and females; and (6) ethnicity of Han origin. (Since the establishment of People's Republic of China, the national government officially identifies 56 distinct ethnic groups according to different dresses, music types, and languages, the largest of which is the Han Chinese. Everybody in China belongs to only one ethnic group that is marked on his/her identity card. Different ethnic groups mean different cultures and cognitive abilities. The patients in the present study all belonged to Han Chinese within three generations. In this way, the interference of ethnic groups could be reduced.) Patients were excluded if they fell into any of the following categories: (1) having a history or presence of any severe unstable systemic disease; (2) having a history of organic brain disease, cerebral trauma, seizure disorder, mental retardation, or MRI evidence of structural brain abnormalities; and (3) being pregnant, lactating, or planning to be pregnant within 6 months.

Volunteers through advertising served as normal controls. None of the controls had a family history of mental disorder or a medical history of somatic or organic brain disease, cerebral trauma, severe mental retardation, or MRI evidence of structural brain abnormalities. All volunteers accepted MRI performed by experienced professional staff in the First Affiliated Hospital of Medical School of Zhejiang University using the Philips Magnetic Resonance Imaging Systems Achieva 3.0T TX (Philips Healthcare, Netherlands). The system was tested for data stability before use. MRI was completed within 1 week after neuropsychological tests. The age and education level of the controls were matched to those of the patients. The controls were of the same ethnic Han origin.

The study was approved by the ethics committee of the First Affiliated Hospital of Medical School of Zhejiang University, and conducted in accordance with the Code of Ethics of the World Medical Association (Declaration of Helsinki). All subjects and their legal guardians (applicable if participants were less than 16 years old) provided written informed consent before participating in the study.

The tests were completed for all subjects by fully trained psychiatrists with consistent training courses in the First
Affiliated Hospital of Medical School of Zhejiang University. The inter-rater agreement of PANSS, WCST, continuous performance test (CPT), TMT and Stroop color-word test (SCWT) was measured, and the kappa coefficient was $0.80,0.82,0.78,0.83$, and 0.84 , respectively. To reduce errors, all items were assessed in the morning and neuropsychological tests were administered first. To minimize the potential of order effects, the sequence of the neuropsychological tests was randomized. The tests usually lasted $1-2 \mathrm{~h}$ and were completed within 1 day for healthy people and two consecutive days for patients. Clinical assessments were rated on the same days as the aforementioned neuropsychological tests.

\section{Neuropsychological assessments}

Four neuropsychological assessments including WCST, CPT, TMT, and SCWT were used in all 149 subjects $(92$ patients with first-episode schizophrenia and 57 healthy controls). All of them were typical tests for patients with schizophrenia in evaluating neurocognitive deficits standing for executive function; sustained, visual, and selective attention; and so forth. In this study, standard Chinese versions of neuropsychological assessments were used, and the rater was blinded to grouping.

\section{Wisconsin card sorting test (modified version)}

The WCST was a cognitive task that predominantly assessed executive function because of its reported sensitivity to frontal lobe dysfunction [31]. Subjects were asked to sort 48 cards on the basis of three possible categories (color, number, and shape). After six consecutive correct responses, the subjects were asked to change the sorting principle to another category. The test ended when the subjects completed all six categories correctly or used 48 cards. The total trials (TT), correct trials (CT), total number of errors (TE), PE, random errors (RE), and number of CC were recorded [32].

\section{Continuous performance test}

The CPT measured the subjects' sustained and selective attention. It was divided into three parts: (1) different numbers appeared on the screen one by one, and the mouse was clicked when "4" appeared; (2) eight numbers appeared on the screen at the same time, and the mouse was clicked when "4" appeared; and (3) eight numbers appeared on the screen at the same time, and the mouse was clicked when "7" appeared. Each number remained on the screen for $150 \mathrm{~ms}$, and the interval time was $550 \mathrm{~ms}$. The subjects were asked to finish all the three parts. The correct trials of three parts (CPT 1, CPT 2, and $\mathrm{CPT} 3$ ) and the perseverative errors in part 3 (PE of CPT 3) were recorded [33]. 


\section{Trail making test}

The TMT was a neuropsychological test of visual attention and task switching, which provided information about visual search speed, processing speed, mental flexibility, and executive functioning. It consisted of two parts in which the subjects were instructed to connect a set of 25 dots as fast and accurately as possible. In part 1 of TMT, the subjects were required to quickly draw lines to connect consecutively numbered circles. In part 2 , the subjects were asked to alternately combine numbers with different colors in ascending order [34]. The task completion was measured in seconds and recorded as TMT 1 and TMT 2, respectively, for parts 1 and 2. TMT 1 was used to examine cognitive processing speed, while TMT 2 referred to executive functioning [35].

\section{Stroop color-word test}

The SCWT, also called Stroop test, measured selective attention, cognitive flexibility, and processing speed. It was used as a tool in evaluating executive functions. In step 1 of SCWT (SCWT 1), the subjects were asked to read out three black words as fast as possible, which stood for a certain color. Then, in step 2 (SCWT 2), they were instructed to tell the color of three color parcels as fast as possible. Finally, in step 3 (SCWT 3), they were required to tell the color of three color words as fast as possible, while each color was different from the word's meaning. The performance for each condition was calculated from the processing time per item in seconds. The reaction time (RT of SCWT) difference in part 3 relative to part 2 was called the "interference" effect [36].

\section{Clinical assessments}

The PANSS [37] was used to assess the clinical symptom severity in 92 patients with first-episode schizophrenia in the time of the neuropsychological tests. The PANSS was a 30-item medical scale, originally grouped into 3 parts, including 7 positive symptoms (i.e., delusions, conceptual disorganization, hallucinations, hyperactivity, grandiosity, suspiciousness/persecution, and hostility), 7 negative symptoms (i.e., blunted affect, emotional withdrawal, poor rapport, passive/apathetic social withdrawal, difficulty in abstract thinking, lack of spontaneity and flow of conversation, and stereotyped thinking) and 16 general psychopathology items (i.e., somatic concern, anxiety, guilt feelings, tension, mannerisms and posturing, depression, motor retardation, uncooperativeness, unusual thought content, disorientation, poor attention, lack of judgment and insight, disturbance of volition, poor impulse control, preoccupation, and active social avoidance) that assessed the degree of psychopathology on a number of symptomatic domains. Each item was rated from 1 (no evidence) to 7 (extreme) based on objective criteria [37]. In this study, standard Chinese versions of PANSS were used to evaluate symptoms of 92 patients with first-episode schizophrenia.

In recent years, the five-factor model has been widely used in different schizophrenia research areas, including cognitive function [14] and insight [38]. With the outcome of principal component analysis and reliability analysis, SI Tianmei suggested that the structure, validity, and reliability of PANSS (Chinese version) were acceptable, and put forward a five-factor model of Chinese-version PANSS, including negative, positive, excitement-hostility, anxiety depression, and cognitive defect factors [39]. In the study, the aforementioned five-factor model was used.

\section{Statistical analyses}

All data were expressed as means \pm standard deviation. An unpaired $t$ test and a chi-square test were used to compare the general conditions and neuropsychological assessment outcomes between patients and healthy people. The relationship between general conditions and neuropsychological assessments of patients was computed using a correlational analysis. A partial correlation was determined to control the potential influence of education level on the relationship between the negative factor scores and neuropsychological assessments. The difference in correlation coefficients between adolescent and adult patients with first-episode schizophrenia was computed by a Fisher's $Z$ test. The false discovery rate was used to control the inflated Type I error rate. To get convincing results, all statistical tests were two-tailed and a $p$-value $<.05$ was considered to be significant.

All analyses were carried out using the Statistical Package for the Social Sciences (SPSS) version 18.0 for Windows (IBM, IL, USA).

\section{Results}

Demographics, neuropsychological assessments, and clinical assessments

No significant difference was found in age $(t=-.697$, $p=.487)$, education level $(t=-1.832, p=.070)$, or gender $\left(\chi^{2}=.129, p=.625\right)$ between patients with first-episode schizophrenia and healthy people.

In the WCST, the mean scores of CT and CC for patients with schizophrenia were lower than those of controls, while the mean scores of TE, PE, and RE for patients with schizophrenia were higher than those of controls (all $p$-values $<.01)$. However, no significant difference was observed in TT $(t=.763, p=.447)$.

The results of TMT and SCWT were similar to those of WCST. Compared with the controls, patients with schizophrenia spent more time in TMT $1(t=2.460$, 
$p=.015)$, TMT $2(t=2.777, p=.006)$, SCWT $2(t=6.574$, $p<.001)$, SCWT $3(t=7.867, p<.001)$ and RT of SCWT $(t=5.000, p<.001)$. In the CPT, patients with first-episode schizophrenia got a higher mean score of PE of CPT 3 compared with healthy people $(t=2.022, p=.045)$. The details are displayed in Table 1.

The mean score of CPT 2 for adolescent patients with first-episode schizophrenia was lower than that of adults $(t=-2.064, p=.043)$. However, no other significant difference was noted in clinical or neuropsychological assessments between adolescent and adult patients with firstepisode schizophrenia. The details are displayed in Table 2.

\section{Correlational analysis between clinical assessments} and neuropsychological assessments in patients with first-episode schizophrenia

Using the five-factor model of PANSS, the negative factor scores were computed using an average of nine items (i.e., Blunted Affect, Emotional Withdrawal, Poor Rapport,
Passive/Apathetic Social Withdrawal, Lack of Spontaneity and Flow of Conversation, Motor Retardation, Disturbance of Volition, Preoccupation, and Active Social Avoidance). Pearson correlational analysis was performed to assess the relationship between general conditions, negative symptoms, and neuropsychological assessments outcomes.

Considering the general conditions, significant correlations were found between the educational level and neuropsychological assessments on nine items, especially on TMT $1(r=-.462, p<.001)$, TMT $2(r=-.473, p<.001)$, SCWT $1(r=-.406, p<.001)$, and SCWT $2(r=-.432$, $p<.001)$. In addition, it has been reported in a previous study that sustained attention, ideation fluency, cognitive flexibility, and visuospatial memory had a positive correlation with education in deficit schizophrenia [40]. Partial correlation analysis was used to get rid of the interference. With the variable of education level controlled, persuasive results were obtained. In total, significant correlations were observed between the negative

Table 1 Demographic, neuropsychological assessments and clinical assessments outcomes of first-episode schizophrenics and healthy people

\begin{tabular}{|c|c|c|c|c|c|}
\hline & & First-episode schizophrenics ( $N=92)$ & Healthy people $(N=57)$ & $t / x^{2}$ & $P$-value \\
\hline \multicolumn{2}{|c|}{ Age (years) } & $22.86 \pm 7.82$ & $23.84 \pm 8.69$ & -.697 & .487 \\
\hline \multicolumn{2}{|c|}{ Education level (years) } & $10.77 \pm 3.01$ & $11.77 \pm 3.38$ & -1.832 & .070 \\
\hline \multicolumn{2}{|c|}{ Duration of illness (months) } & $12.26 \pm 18.37$ & None & None & None \\
\hline \multicolumn{2}{|c|}{ Gender (male/total) } & $36 / 92$ & $24 / 57$ & .129 & .625 \\
\hline \multirow[t]{4}{*}{ PANSS } & $\mathrm{T}$ & $84.18 \pm 16.71$ & None & None & None \\
\hline & $P$ & $23.88 \pm 7.37$ & None & None & None \\
\hline & N & $19.30 \pm 8.12$ & None & None & None \\
\hline & G & $41.00 \pm 8.32$ & None & None & None \\
\hline \multirow[t]{6}{*}{ WCST } & $\Pi$ & $47.34 \pm 2.73$ & $47.04 \pm 2.08$ & .763 & .447 \\
\hline & $\mathrm{CT}$ & $25.42 \pm 10.47$ & $33.91 \pm 7.30$ & -5.821 & $<.001$ \\
\hline & $\mathrm{TE}$ & $21.80 \pm 10.56$ & $12.77 \pm 8.18$ & 5.846 & $<.001$ \\
\hline & PE & $13.82 \pm 7.54$ & $7.56 \pm 4.99$ & 6.089 & $<.001$ \\
\hline & RE & $7.96 \pm 5.00$ & $5.49 \pm 3.94$ & 3.342 & .001 \\
\hline & CC & $2.87 \pm 2.06$ & $4.68 \pm 1.68$ & -5.872 & $<.001$ \\
\hline \multirow[t]{4}{*}{ CPT } & CPТ 1 & $11.01 \pm 2.27$ & $11.18 \pm .80$ & -.635 & .527 \\
\hline & CPT 2 & $11.63 \pm 3.33$ & $12.12 \pm 1.65$ & -1.201 & .232 \\
\hline & СРТ 3 & $11.95 \pm 2.99$ & $12.23 \pm 1.28$ & -.795 & .428 \\
\hline & PE of CPT 3 & $.84 \pm 1.75$ & $.37 \pm 1.08$ & 2.022 & .045 \\
\hline \multirow[t]{2}{*}{ TMT } & TMT 1 & $58.13 \pm 34.06$ & $43.56 \pm 35.77$ & 2.460 & .015 \\
\hline & TMT 2 & $114.31 \pm 62.47$ & $84.51 \pm 64.39$ & 2.777 & .006 \\
\hline \multirow[t]{4}{*}{ SCWT } & SCWT 1 & $50.82 \pm 15.19$ & $44.96 \pm 21.87$ & 1.772 & .080 \\
\hline & SCWT 2 & $90.16 \pm 33.32$ & $62.51 \pm 17.90$ & 6.574 & $<.001$ \\
\hline & SCWT 3 & $143.27 \pm 49.19$ & $97.51 \pm 22.82$ & 7.687 & $<.001$ \\
\hline & RT of SCWT & $53.10 \pm 29.27$ & $35.00 \pm 14.72$ & 5.000 & $<.001$ \\
\hline
\end{tabular}

Values are the means \pm SD. All $P$-values were compared with Healthy people analyzed by an unpaired $t$-test

$T$ PANSS total scores, $P$ positive syndrome scale scores, $N$ negative syndrome scale scores, $G$ general psychopathology syndrome scale scores, $\pi$ total trials, $C T$ correct trials, $T E$ total number of errors, $P E$ perseverative errors, $R E$ random errors, $C C$ the number of completed categories, $P E$ of $C P T 3$ perseverative errors of CPT 3, RT of SCWT reaction time of SCWT; 
Table 2 Demographic, neuropsychological assessments and clinical assessments outcomes of adolescent and adult first-episode schizophrenics

\begin{tabular}{|c|c|c|c|c|c|}
\hline \multirow{2}{*}{\multicolumn{2}{|c|}{ Age (years) }} & Adolescent first-episode schizophrenics $(N=33)$ & Adult first-episode schizophrenics $(N=59)$ & $\mathrm{t}$ & $P$-value \\
\hline & & 15.48 & 25.02 & -10.107 & $<.001$ \\
\hline \multicolumn{2}{|c|}{ Education level (years) } & 9.61 & 11.42 & -3.518 & .001 \\
\hline \multicolumn{2}{|c|}{ Duration of illness (months) } & $9.67 \pm 1.50$ & $11.42 \pm 3.43$ & -1.182 & .240 \\
\hline \multirow[t]{4}{*}{ PANSS } & T & $85.30 \pm 18.04$ & $83.56 \pm 16.04$ & .462 & .645 \\
\hline & P & $23.33 \pm 7.21$ & $24.19 \pm 7.50$ & -.537 & .593 \\
\hline & N & $20.45 \pm 10.26$ & $18.66 \pm 6.65$ & .904 & .371 \\
\hline & G & $41.52 \pm 8.70$ & $40.71 \pm 8.16$ & .434 & .666 \\
\hline \multirow[t]{6}{*}{ WCST } & $\pi$ & $47.64 \pm 1.11$ & $47.17 \pm 3.30$ & .990 & .325 \\
\hline & CT & $24.06 \pm 9.62$ & $26.19 \pm 10.92$ & -.968 & .336 \\
\hline & TE & $23.27 \pm 10.15$ & $20.98 \pm 10.78$ & 1.015 & .314 \\
\hline & PE & $14.64 \pm 6.28$ & $13.36 \pm 8.18$ & .840 & .403 \\
\hline & RE & $8.55 \pm 5.10$ & $7.63 \pm 4.95$ & .837 & .406 \\
\hline & CC & $2.64 \pm 2.07$ & $3.00 \pm 2.05$ & -.810 & .421 \\
\hline \multirow[t]{4}{*}{ CPT } & CPT 1 & $10.79 \pm 2.30$ & $11.14 \pm 2.25$ & -.700 & .486 \\
\hline & CPT 2 & $10.67 \pm 3.45$ & $12.17 \pm 3.16$ & -2.064 & .043 \\
\hline & CPT 3 & $11.61 \pm 3.65$ & $12.14 \pm 2.57$ & -.738 & .464 \\
\hline & PE of CPT 3 & $.61 \pm 1.48$ & $.97 \pm 1.88$ & -1.013 & .314 \\
\hline \multirow[t]{2}{*}{ TMT } & TMT 1 & $57.85 \pm 30.69$ & $58.28 \pm 36.06$ & -.061 & .951 \\
\hline & TMT 2 & $104.68 \pm 48.052$ & $119.69 \pm 69.05$ & -1.223 & .225 \\
\hline \multirow[t]{4}{*}{ SCWT } & SCWT 1 & $50.50 \pm 16.44$ & $50.99 \pm 14.59$ & -.145 & .885 \\
\hline & SCWT 2 & $88.25 \pm 30.03$ & $91.23 \pm 35.23$ & -.430 & .669 \\
\hline & SCWT 3 & $134.98 \pm 33.04$ & $147.90 \pm 55.98$ & -1.391 & .168 \\
\hline & RT of SCWT & $46.74 \pm 19.66$ & $56.66 \pm 33.09$ & -1.804 & .075 \\
\hline
\end{tabular}

Values are the means \pm SD. All $P$-values were compared with Healthy people analyzed by an unpaired $t$-test

$T$ PANSS total scores, $P$ positive syndrome scale scores, $N$ negative syndrome scale scores, $G$ general psychopathology syndrome scale scores, $T T$ total trials, $C T$ correct trials, TE total number of errors, PE perseverative errors, RE random errors, CC the number of completed categories, $P E$ of $C P T 3$ perseverative errors of CPT 3, RT of SCWT reaction time of SCWT;

factor scores and neuropsychological assessments on 12 items (i.e., CT, TE, PE, RE and CC of WCST; CPT 1; TMT 1 and TMT 2; SCWT 1, SCWT 2, SCWT 3, and RT of SCWT). Moreover, the negative factor score was moderately correlated to SCWT $1(r=.410, p<.001)$ and SCWT $3(r=.409, p<.001)$. The details are displayed in Table 3.

\section{Comparison of adolescent and adult patients with first-episode schizophrenia}

In both adolescent and adult patients with schizophrenia, the negative factor score was correlated with more time spent in part $1(r=.527, p=.002 ; r=.342, p=.009)$, part 2 $(r=.400, p=.023 ; r=.365, p=.005)$, and part $3(r=.552$, $p=.001 ; r=.452, p<.001)$ of SCWT, a lower mean score of CT $(r=-.486, p=.005 ; r=-.313, p=.017)$, higher mean scores of TE $(r=.489, p=.005 ; r=.297, p=.024)$, and RE of WCST $(r=.488, p=.005 ; r=.310, p=.018)$. No significant difference was found in the aforementioned items of correlation coefficients (all $p$-values $>.05$ ) between the two groups. The details are displayed in Table 4.

In adolescent patients with schizophrenia, the negative factor score was strongly correlated with more time spent in TMT $1(r=.646, p<.001)$ and TMT $2(r=.663$, $p<.001)$, and moderately or weakly correlated with a higher mean score of PE $(r=.425, p=.015)$ of WCST and lower mean scores of CPT $2(r=-.425, p=.015)$. However, no correlation between the aforementioned items could be found in adult patients.

The negative factor score was weakly correlated with a lower mean score of CPT $3(r=-.385, p=.030)$ in adolescent patients with schizophrenia, while it was weakly correlated with a higher mean score of CPT $3(r=.303$, $p=.021)$ in adults. The details are displayed in Table 5.

\section{Discussion}

The most important finding in the present study was significant correlations between the negative factor scores and most neuropsychological assessments in patients with 
Table 3 The correlation and partial correlation (negative factor) matrix of general conditions, negative symptoms and neuropsychological assessments outcomes on first-episode schizophrenics

\begin{tabular}{|c|c|c|c|c|c|c|}
\hline & & Age & Age of onset & Education level & Duration of illness & Negative factor \\
\hline \multirow[t]{6}{*}{ WCST } & $\pi$ & -.032 & -.030 & .004 & -.011 & -.010 \\
\hline & CT & .044 & -.028 & $.276^{* *}$ & -.075 & $-.381^{* *}$ \\
\hline & TE & -.045 & .028 & $-.271^{* *}$ & .078 & $.381^{* *}$ \\
\hline & PE & -.034 & .043 & $-.257^{*}$ & .086 & $.272^{* *}$ \\
\hline & RE & -.033 & -.001 & -.159 & .002 & $.397^{* *}$ \\
\hline & $\mathrm{CC}$ & -.028 & -.084 & $.283^{* *}$ & -.044 & $-.290^{* *}$ \\
\hline \multirow[t]{4}{*}{ CPT } & CPT 1 & $.242^{*}$ & .179 & .141 & $.251^{*}$ & $-.230^{*}$ \\
\hline & CPT 2 & .187 & .192 & -.044 & .148 & -.178 \\
\hline & CPT 3 & .040 & .002 & .056 & -.090 & -.080 \\
\hline & PE of CPT 3 & .051 & .099 & -.007 & .024 & -.045 \\
\hline \multirow[t]{2}{*}{ TMT } & TMT 1 & -.009 & .175 & $-.462^{* *}$ & .016 & $.306^{* *}$ \\
\hline & TMT 2 & .043 & $.222^{*}$ & $-.473^{* *}$ & .014 & $.293^{* *}$ \\
\hline \multirow[t]{4}{*}{ SCWT } & SCWT 1 & .087 & .156 & $-.406^{* *}$ & .055 & $.410^{* *}$ \\
\hline & SCWT 2 & -.067 & .087 & $-.432^{* *}$ & .072 & $.331^{* *}$ \\
\hline & SCWT 3 & -.063 & -.013 & $-.206^{*}$ & -.104 & $.409^{* *}$ \\
\hline & RT of SCWT & -.029 & -.120 & .145 & $-.256^{*}$ & $.335^{* *}$ \\
\hline
\end{tabular}

False Discovery Rate: $p(\mathrm{CT})<p$ (TE) $<p$ (RE) $<p$ (SCWT 1) $<p$ (SCWT 3) $<p$ (SCWT 2$)<p$ (RT of SCWT) $<p$ (TMT 1) $<p$ (CC) $<p$ (TMT 2) $<p$ (PE) $<p$ (CPT 1$)<.05<$ $p$ (CPT 2) $<p$ (CPT 3) $<p$ (PE of CPT 3) $<p$ (TT), while $p$ (CPT 1) $=.028<.05 * 12 / 16=.0375$

$|\mathrm{r}| \in[.8,1]$ means highly strong correlation, $|\mathrm{r}| \in[.6, .8)$ means strong correlation, $|\mathrm{r}| \in[.4, .6)$ means moderate correlation, $|\mathrm{r}| \in[.2,4)$ means weak correlation, $|\mathrm{r}| \in[0, .2)$ means very weak correlation or no correlation

$\pi$ total trials, $C T$ correct trials, $T E$ total number of errors, $P E$ perseverative errors, $R E$ random errors, $C C$ the number of completed categories; $P E$ of $C P T 3$ perseverative errors of CPT 3, RT of SCWT reaction time of SCWT

${ }^{*} P<.05,{ }^{* *} P<.01$, the significance of the correlation coefficient

first-episode schizophrenia, with stronger correlations in adolescent patients. Most previous findings [22, 23] indicated that more attention should be paid to patients with first-episode schizophrenia who are drug naïve, to reduce the interference of drugs with symptoms and neurocognitive functions, and also to the differences between adolescence and adults.

On one hand, patients diagnosed with schizophrenia showed neurocognitive deficits in most assessments compared with healthy people, including executive function; visual search and processing speed; sustained, selective, and visual attention; and mental and cognitive flexibility.

Table 4 The comparison of correlation coefficients between adolescent and adult first-episode schizophrenics

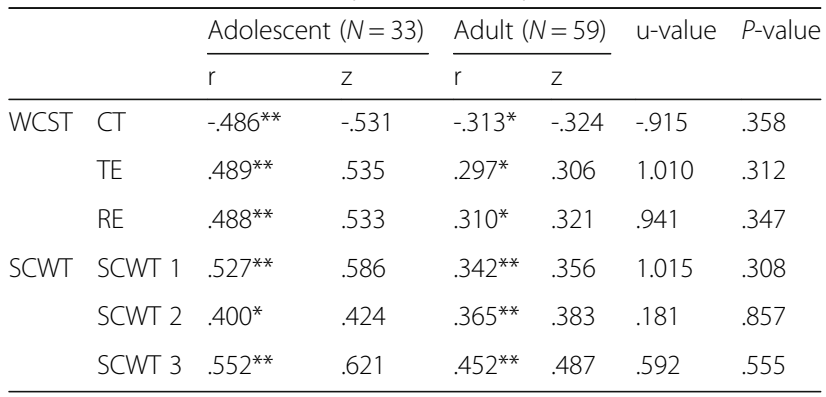

$C T$ correct trials, $T E$ total number of errors, RE random errors

${ }^{*} P<.05,{ }^{* *} P<.01$, the significance of the correlation coefficient
The present results were similar to many previous reports in the literature, which suggested that patients with schizophrenia showed deficits in executive function [31], visual attention, visual search speed, processing speed, mental flexibility [35] and selective attention [36]. That is to say, schizophrenia is a mental disorder that affects one's neurocognitive function [2]. Some brain imaging examinations such as magnetic resonance tomography and positron emission tomography-computed tomography supported that abnormal functional areas in the brain of patients with schizophrenia may usually appear in the frontal lobes and temporal lobes [4]. The frontal lobe plays an important role in executive function, including the ability to forecast future outcomes resulting from present decisions, to determine better or worse choices, to deal with social emergencies, and to distinguish similarities and differences. The functions of temporal lobes cover reserving visual memories, processing sensory input, understanding language, keeping new memories and emotions, and extending in meaning. Some correlations may exist between abnormal activity of massive brain regions and neurocognitive function deficits in patients with schizophrenia [2]. Thus far, imaging examinations have been completed in some of these 149 subjects, and the details will be discussed in future studies. On the other hand, No significant difference was observed 
Table 5 The correlation matrix of negative factor scores and the neuropsychological test measures on adolescent and adult first-episode schizophrenics

\begin{tabular}{|c|c|c|c|c|}
\hline & & \multicolumn{3}{|c|}{ Negative factor scores } \\
\hline & & Total & Adolescent & Adult \\
\hline \multirow[t]{6}{*}{ WCST } & $\pi$ & -.010 & .050 & -.059 \\
\hline & CT & $-.381^{* *}$ & $-.486^{* *}$ & $-.313^{*}$ \\
\hline & TE & $.381^{* *}$ & $.489^{* *}$ & $.297^{*}$ \\
\hline & PE & $.272^{* *}$ & $.425^{*}$ & .199 \\
\hline & RE & $.397^{* *}$ & $.488^{* *}$ & $.310^{*}$ \\
\hline & CC & $-.290^{* *}$ & -.352 & -.214 \\
\hline \multirow[t]{4}{*}{ CPT } & CPТ 1 & $-.230^{*}$ & -.368 & -.094 \\
\hline & CPT 2 & -.178 & $-.425^{*}$ & .081 \\
\hline & CPT 3 & -.080 & $-.385^{*}$ & $.303^{*}$ \\
\hline & PE of CPT 3 & -.045 & .221 & -.150 \\
\hline \multirow[t]{2}{*}{ TMT } & TMT 1 & $.306^{* *}$ & $.646^{* *}$ & .101 \\
\hline & TMT 2 & $.293^{* *}$ & $.663^{* *}$ & .173 \\
\hline \multirow[t]{4}{*}{ SCWT } & SCWT 1 & $.410^{* *}$ & $.527^{* *}$ & $.342^{* *}$ \\
\hline & SCWT 2 & $.331^{* *}$ & $.400^{*}$ & $.365^{* *}$ \\
\hline & SCWT 3 & $.409^{* *}$ & $.552^{* *}$ & $.452^{* *}$ \\
\hline & RT of SCWT & $.335^{* *}$ & .318 & $.412^{* *}$ \\
\hline
\end{tabular}

Adolescent False Discovery Rate: $p$ (TMT 1) $<p$ (TMT 2) $<p$ (SCWT 3) $<p$ (SCWT 1) $<p$ (CT) $<p$ (TE) $<p$ (RE) $<p$ (PE) $<p$ (CPT 2) $<p$ (SCWT 2) $<p$ (CPT 3) $<p$ (CPT 1) $<$ $p$ (CC) $<.05<p$ (RT of SCWT) $<p$ (PE of CPT 3) $<p$ (TT), while $p$ (CPT 3$)=.03$ $<.05^{*} 11 / 16=.034$ and $p$ (CPT 1$)=.038>.05^{*} 12 / 16=.0375$. So CC and CPT 1 are not significant

Adult False Discovery Rate: $p$ (SCWT 3) $<p$ (RT of SCWT) $<p$ (SCWT 2) $<p$ (SCWT 1) $<p$ (CT) $<p$ (RE) $<p$ (CPT 3) $<p$ (TE) $<.05<p$ (CC) $<p$ (PE) $<p$ (TMT 2) $<p$ (PE of CPT 3) $<p$ (TMT 1) $<p$ (CPT 1) $<p$ (CPT 2) $<p$ (TT), while $p(\mathrm{TE})=.024<.05 * 8 / 16=.025$

$|r| \in[.8,1]$ means highly strong correlation, $|r| \in[.6,8)$ means strong correlation, $|r| \in[.4,6)$ means moderate correlation, $|r| \in[.2,4)$ means weak correlation, $|r| \in[0,2)$ means very weak correlation or no correlation

$\pi$ total trials, $C T$ correct trials, TE total number of errors, PE perseverative errors, $R E$ random errors, $C C$ the number of completed categories, PE of CPT 3 perseverative errors of CPT 3, RT of SCWT reaction time of SCWT

${ }^{*} P<.05,{ }^{*} P<.01$, the significance of the correlation coefficient

in clinical or neuropsychological assessments between adolescent and adult patients with first-episode schizophrenia, but some previous studies showed that adolescent patients with schizophrenia performed worse than adult patients in tasks of working memory, language, and motor function [21].

Significant correlations were obtained between negative symptoms and most neuropsychological assessments (CT, TE, PE, RE, and CC of WCST; CPT 1; TMT 1 and TMT 2; SCWT 1, SCWT 2, SCWT 3, and RT of SCWT) in patients with first-episode schizophrenia. It meant that the severity of negative symptoms was correlated with the deficits of executive function, sustained, selective, and visual attention, visual search and processing speed, and mental and cognitive flexibility in patients with schizophrenia [32, $33,35,36]$. A previous similar study showed that negative symptoms were associated with neurocognitive function deficits involving executive function, sustained attention function [22], and cognitive function [23].

Most importantly, the present findings suggested closer correlations between the negative factor scores and some neuropsychological assessments outcomes in adolescent patients with first-episode schizophrenia, including more time spent in TMT 1 and TMT 2, fewer times of CPT 2, and a higher mean score of PE of WCST, compared with adult patients. Therefore, the severity of negative symptoms in adolescent patients with schizophrenia was more related to their neurocognitive deficits of visual, selective, and sustained attention; visual search and processing speed; mental flexibility; and executive function [36-38]. Some of the latest studies regarded schizophrenia as a collection of "neurodevelopmental disorders," because it nearly emerged in late adolescence or early adulthood when the prefrontal cortex was still developing [2, 28-30]. The present results partly support the conclusion. Since adolescence is a key period of neurological and psychological development, earlier schizophrenia symptoms and neurocognitive deficits may both originate from earlier neurodevelopmental problems in adolescent patients with first-episode schizophrenia.

Hence, the negative symptoms of adolescent patients with schizophrenia are more closely related to their neurocognitive deficits, compared with adult patients with first-episode schizophrenia. For physicians, the negative symptom scores of PANSS become more important in evaluating neurocognitive deficits of adolescent patients with first-episode schizophrenia. Because it is easy for psychiatrists to obtain symptom characteristics from psychiatric examinations, PANSS scores, and description of patients or their family members, the physicians can view the severity of negative symptoms as a factor that infers neurocognitive deficits in patients with first-episode schizophrenia, especially adolescents. The evaluation of neurocognitive functions is complicated and timeconsuming. First-visit adolescent outpatients with obvious negative symptoms may suggest serious neurocognitive deficits. Furthermore, the severity of negative symptoms can be widely used in evaluating the therapeutic effect.

The present study had several limitations. (1) The sample size was small. (2) The ratio of male and female participants was different between groups. (3) The study did not contain any follow-up data. (4) The study lacked neuroimaging examinations. (5) The cutoff age remained unclear. In this study, a total of 92 patients with schizophrenia were divided into two groups-33 adolescent patients with first-episode schizophrenia (whose age and age of onset were less than 18 years) and 59 adult patients with firstepisode schizophrenia (whose age and age of onset were equal to or more than 18 years), which meant the cutoff age was 18 years. However, recent research has regarded schizophrenia to comprise four stages (i.e., stage 1: 
$<12$ years, risk stage; stage 2: $12-18$ years, prodromal stage; stage 3: 18-24 years, psychosis stage; stage 4: $>24$ years, chronic disabled stage) [2]. Thus, it would be better that more cutoff points are set in future studies.

In summary, clinical negative symptoms are good indexes for evaluating neurocognitive deficits in patients with schizophrenia. In adolescent patients, doctors should pay more attention to negative symptom results of PANSS. Also, more brain imaging data should be collected to support the conclusions. Moreover, longer-term studies with a larger sample size are needed to draw more definite conclusions.

\section{Conclusions}

1. Patients with first-episode schizophrenia show neurocognitive deficits in most neuropsychological assessments compared with healthy people.

2. The severity of negative symptoms in patients with first-episode schizophrenia is related to some of their neurocognitive deficits.

3. Most importantly, this relationship is even closer for adolescent patients with first-episode schizophrenia.

\section{Abbreviations}

CC: Completed categories; CPT: Continuous performance test; CT: Correct trials; PANSS: Positive and negative syndrome scale; PE: Perseverative errors; RE: Random errors; RT: Reaction time; SCWT: Stroop color-word test; TE: Total number of errors; TMT: Trail making test; TT: Total trials; WCST: Wisconsin card sorting test

\section{Acknowledgements}

We'd like to appreciate Yi Shen's statistical advice throughout the entire project and all the subjects who participated in the study.

\section{Funding}

This research was supported by grants 2015BAl13B02 from the China National Clinical research Center for Mental Health Disorders, grants $2010 R 50049$ from the Science and Technology Bureau of Zhejiang Province Key Science and Technology Innovation Project, grants Y201120182 from the Education Bureau of Zhejiang Province, grants 2013RCA017 from Medical Platform Key Talent Plan of Zhejiang Province, grants 2013RCB005, 2014RCA005 from the Department of Health Foundation of Zhejiang Province, and grants 81301158 from National Natural Science Foundation of China.

\section{Availability of data and materials}

Datasets of the current study are saved in Department of Psychiatry, The First Affiliated Hospital, College of Medicine, Zhejiang University. Due to ethical restrictions, data are available from the corresponding author on reasonable request.

\section{Authors' contributions}

YX planed and drafted the study. MLH designed the study, collected the data and advised on modeling strategies. YH analyzed the data and wrote the paper. JBH evaluated the scales. LY, JKC, PBJ, WJX, NW, SHH and HLQ collected the data. MLH and YH contributed equally to this work. All authors read and approved the final manuscript.

\section{Competing interests}

The authors declare that they have no competing interests.

Consent for publication

All subjects in the study have signed consent for publication.

\section{Ethics approval and consent to participate}

The study is approved by Ethics Committee of the First Affiliated Hospital, College of Medicine, Zhejiang University with a Reference Number of 2013-202, and conducted in accordance with the Code of Ethics of the World Medical Association (Declaration of Helsinki).

All subjects and their legal guardians (applicable if participants were less than 16 years old) provided written informed consent before participating in the study.

\section{Highlights}

1. First-episode schizophrenia patients show neurocognitive deficits in most neuropsychological assessments compared with healthy people.

2. There are significant correlations between the negative factor and neuropsychological assessments in first-Episode Schizophrenia patients.

3. With the variable of education level been controlled, the negative factor of adolescent first-episode schizophrenia patients was strongly correlated to more time spent in part $1(r=0.646, P<0.001)$ and part $2(r=0.663$, $P<0.001$ ) of Trail Making Test, and it was moderately correlated to more perseverative errors $(r=0.425, P=0.015)$ of Wisconsin Card Sorting Test and fewer correct trials $2(r=-0.425, P=0.015)$ of Continuous Performance Test. However, no correlation could be found in adult patients in the above items

\section{Author details}

1Department of Psychiatry, The First Affiliated Hospital, College of Medicine, Zhejiang University, The Key Laboratory of Mental Disorder's Management of Zhejiang Province, No. 79, Qingchun Road, Hangzhou 310003, China.

2Department of General Surgery, The Second Affiliated Hospital, College of Medicine, Zhejiang University, No. 88, Jiefang Road, Hangzhou 310000, China. ${ }^{3}$ Department of Anesthesiology and Pain, Hang Zhou First People's Hospital, No. 261, Huansha Road, Hangzhou 310006, China.

Received: 24 August 2015 Accepted: 24 September 2016

Published online: 06 October 2016

\section{References}

1. van Os J, Kapur S. Schizophrenia. Lancet. 2009;374(9690):635-45.

2. Insel TR. Rethinking schizophrenia. Nature. 2010;468(7321):187-93.

3. Kirkbride JB, Fearon $P$, Morgan $C$, et al. Neighbourhood variation in the incidence of psychotic disorders in Southeast London. Soc Psychiatry Psychiatr Epidemiol. 2007:42(6):438-45.

4. Kircher TT, Thienel R. Functional brain imaging of symptoms and cognition in schizophrenia. Prog Brain Res. 2005;150:299-308.

5. MacPherson M. Psychological causes of schizophrenia. Schizophr Bull. 2009;35(2):284-6.

6. Khan ZU, Martin-Montanez E, Muly EC. Schizophrenia: causes and treatments. Curr Pharm Des. 2013;19(36):6451-61.

7. Jerrell JM, Hrisko S. A comparison of the PANSS Pentagonal and Van Der Gaag 5-factor models for assessing change over time. Psychiatry Res. 2013; 207(1-2):134-9.

8. Lancon C, Aghababian V, Llorca PM, Auquier P. Factorial structure of the Positive and Negative Syndrome Scale (PANSS): a forced five-dimensional factor analysis. Acta Psychiatr Scand. 1998;98(5):369-76.

9. Lancon C, Auquier P, Nayt G, Reine G. Stability of the five-factor structure of the Positive and Negative Syndrome Scale (PANSS). Schizophr Res. 2000;42(3):231-9.

10. Van den Oord EJ, Rujescu D, Robles JR, et al. Factor structure and external validity of the PANSS revisited. Schizophr Res. 2006;82(2-3):213-23.

11. Citrome L, Meng $X$, Hochfeld M. Efficacy of iloperidone in schizophrenia: a PANSS five-factor analysis. Schizophr Res. 2011;131(1-3):75-81.

12. Jerrell JM, Hrisko S. Utility of Two PANSS 5-Factor Models for Assessing Psychosocial Outcomes in Clinical Programs for Persons with Schizophrenia. Schizophr Res Treatment. 2013;2013:705631.

13 Wallwork RS, Fortgang R, Hashimoto R, Weinberger DR, Dickinson D. Searching for a consensus five-factor model of the Positive and Negative Syndrome Scale for schizophrenia. Schizophr Res. 2012;137(1-3):246-50.

14 Rodriguez-Jimenez R, Bagney A, Mezquita L, et al. Cognition and the fivefactor model of the Positive and Negative Syndrome Scale in schizophrenia. Schizophr Res. 2013;143(1):77-83.

15 Tsai J, Lysaker PH, Vohs JL. Negative symptoms and concomitant attention deficits in schizophrenia: associations with prospective 
assessments of anxiety, social dysfunction, and avoidant coping J Ment Health. 2010;19(2):184-92.

16 Hunter R, Barry S. Negative symptoms and psychosocial functioning in schizophrenia: neglected but important targets for treatment. Eur Psychiatry. 2012;27(6):432-6.

17 Makinen J, Miettunen J, Isohanni M, Koponen H. Negative symptoms in schizophrenia: a review. Nord J Psychiatry. 2008;62(5):334-41.

18 Smith T, Weston C, Lieberman J. Schizophrenia (maintenance treatment). Am Fam Physician. 2010;82(4):338-9.

19 White T, Andreasen NC, Nopoulos P, Magnotta V. Gyrification abnormalities in childhood- and adolescent-onset schizophrenia. Biol Psychiatry. 2003;54(4):418-26.

20 Thompson PM, Vidal C, Giedd JN, et al. Mapping adolescent brain change reveals dynamic wave of accelerated gray matter loss in very early-onset schizophrenia. Proc Natl Acad Sci U S A. 2001:98(20):11650-5.

21 White T, Ho BC, Ward J, O'Leary D, Andreasen NC. Neuropsychological performance in first-episode adolescents with schizophrenia: a comparison with first-episode adults and adolescent control subjects. Biol Psychiatry. 2006;60(5):463-71.

22 Basso MR, Nasrallah HA, Olson SC, Bornstein RA. Neuropsychological correlates of negative, disorganized and psychotic symptoms in schizophrenia. Schizophr Res. 1998:31(2-3):99-111.

23 Jimenez-Arriero MA, Rodriguez-Jimenez R, Rubio G, et al. Clinical and neuropsychological differences in schizophrenia according to negative symptom PANSS scores. Eur Psychiatry. 2007;22:S136.

24 Godbout L, Limoges F, Allard I, Braun CMJ, Stip E. Neuropsychological and activity of daily living script performance in patients with positive or negative schizophrenia. Compr Psychiatry. 2007;48(3):293-302.

25 Berman I, Viegner B, Merson A, Allan E, Pappas D, Green Al. Differential relationships between positive and negative symptoms and neuropsychological deficits in schizophrenia. Schizophr Res. 1997;25(1):1-10.

26 Brewer WJ, Edwards J, Anderson V, Robinson T, Pantelis C. Neuropsychological, olfactory, and hygiene deficits in men with negative symptom schizophrenia. Biol Psychiatry. 1996;40(10):1021-31.

27 Martino DJ, Bucay D, Butman JT, Allegri RF. Neuropsychological frontal impairments and negative symptoms in schizophrenia. Psychiatry Res. 2007:152(2-3):121-8.

28 Fatemi SH, Folsom TD. The Neurodevelopmental Hypothesis of Schizophrenia, Revisited. Schizophr Bull. 2009;35(3):528-48.

29 Cornblatt BA, Lencz T, Smith CW, Correll CU, Auther AM, Nakayama E. The schizophrenia prodrome revisited: A neurodevelopmental perspective. Schizophr Bull. 2003;29(4):633-51.

30 Ashe PC, Berry MD, Boulton AA. Schizophrenia, a neurodegenerative disorder with neurodevelopmental antecedents. Prog Neuropsychopharmacol Biol Psychiatry. 2001;25(4):691-707.

31 Greve KW, Stickle TR, Love JA, Bianchini K, Stanford MS. Latent structure of the Wisconsin Card Sorting Test: a confirmatory factor analytic study. Arch Clin Neuropsychol. 2005;20(3):355-64.

32 Nelson HE. A modified card sorting test sensitive to frontal lobe defects. Cortex. 1976;12(4):313-24.

33 Rapisarda A, Kraus M, Tan YW, et al. The continuous performance test, identical pairs: norms, reliability and performance in healthy controls and patients with schizophrenia in Singapore. Schizophr Res. 2014;156(2-3):233-40.

34 Bowie CR, Harvey PD. Administration and interpretation of the Trail Making Test. Nat Protoc. 2006;1(5):2277-81.

35 Tombaugh TN. Trail Making Test A and B: normative data stratified by age and education. Arch Clin Neuropsychol. 2004;19(2):203-14.

36 Stroop JR. Studies of interference in serial verbal reactions. J Exp Psychol. 1935;18:643-62.

37 Kay SR, Fiszbein A, Opler LA. The positive and negative syndrome scale (PANSS) for schizophrenia. Schizophr Bull. 1987:13(2):261-76.

38 Monteiro LC, Silva VA, Louza MR. Insight, cognitive dysfunction and symptomatology in schizophrenia. Eur Arch Psychiatry Clin Neurosci. 2008;258(7):402-5.

39 SI Tianmei YJ, Liang SHU. The Reliability, Validity of PANSS and its Implication. Chin Ment Health J. 2004;18(1):45-7.

40 Yu M, Tang X, Wang X, et al. Neurocognitive Impairments in Deficit and Non-Deficit Schizophrenia and Their Relationships with Symptom Dimensions and Other Clinical Variables. PLoS One. 2015;10(9):e0138357. 